\title{
Correction to: Genetic and epigenetic mechanisms in the development of arteriovenous malformations in the brain
}

\author{
Jaya Mary Thomas ${ }^{1,4}$, Sumi Surendran ${ }^{1}$, Mathew Abraham ${ }^{3}$, Arumugam Rajavelu ${ }^{1,2^{*}}$ \\ and Chandrasekharan C. Kartha ${ }^{1^{*}}$
}

\section{Correction}

Upon publication of the original article [1] the authors noticed that the affiliation Manipal Academy of Higher Education (MAHE), Manipal, Karnataka, India was missing. The addition of this affiliation has been formally noted in this correction article.

\begin{abstract}
Author details
${ }^{1}$ Cardiovascular Disease Biology Program, Rajiv Gandhi Centre for Biotechnology, Poojapura, Thycaud, Thiruvananthapuram, Kerala, India. ${ }^{2}$ Tropical Disease Biology Program, Rajiv Gandhi Centre for Biotechnology, Poojapura, Thycaud, Thiruvananthapuram, Kerala, India. ${ }^{3}$ Department of Neurosurgery, Sree Chitra Tirunal Institute for Medical Sciences \& Technology, Thiruvananthapuram, Kerala, India. ${ }^{4}$ Manipal Academy of Higher Education (MAHE), Manipal, Karnataka, India.
\end{abstract}

Received: 5 October 2018 Accepted: 5 October 2018

Published online: 17 October 2018

\section{Reference}

1. Thomas JM, Surendran S, Abraham M, Rajavelu A, Kartha CC. Genetic and epigenetic mechanisms in the development of arteriovenous malformations in the brain. Clin Epigenetics. 2016;8(1):78.

\footnotetext{
* Correspondence: arajavelu@rgcb.res.in; cckartha@rgcb.res.in

${ }^{1}$ Cardiovascular Disease Biology Program, Rajiv Gandhi Centre for

Biotechnology, Poojapura, Thycaud, Thiruvananthapuram, Kerala, India
} 